\title{
Prevalence of Sexual Dysfunction in Females with and Without Schizophrenia
}

\author{
Pallavi Abhilasha, Mrinalini Reddy, Rajkumar Ramanathan, Thirunavukarasu Manickam, \\ Sivabalan Elangovan*
}

Department of Psychiatry, SRM Medical College \& Research Centre, Tamilnadu, India

Email address:

sivabalanela@gmail.com (S. Elangovan)

${ }^{*}$ Corresponding author

\section{To cite this article:}

Pallavi Abhilasha, Mrinalini Reddy, Rajkumar Ramanathan, Thirunavukarasu Manickam, Sivabalan Elangovan. Prevalence of Sexual Dysfunction in Females with and Without Schizophrenia. American Journal of Psychiatry and Neuroscience.

Vol. 6, No. 3, 2018, pp. 56-60. doi: 10.11648/j.ajpn.20180603.11

Received: June 1, 2018; Accepted: July 3, 2018; Published: August 1, 2018

\begin{abstract}
INTRODUCTION- Schizophrenia is an illness that may influence every aspect of the person's life. Rates of female sexual dysfunction (FSD) in schizophrenia the rates reported have been between 30 and $80 \%$. The aim was to identify attribution (locus of control) and sexual dysfunction among female with schizophrenic disorder and females without schizophrenia and to study and to compare the sexual dysfunction and attribution in schizophrenic and non-schizophrenic women. METHODS- This was a cross sectional, hospital-based study and was approved by the Institutional Ethics Committee of SRM Medical college and health sciences. Women between the ages of 21 to 45 years, diagnosed with schizophrenia by ICD 10 criteria (World Health Organization, 1992) attending the outpatient services, who were under remission, and were in marital relationship for at least six months were approached for the study. Schizophrenia was assessed using PANSS while sexual dysfunction in females was assessed using female sexual functioning index. Sexual attribution was assessed by using Rotter's locus of control. RESULTS- 45 women belonging to cases had sexual dysfunction as assessed by FSFI whilst 13 had had scored for sexual dysfunction is the control group. 5 in the cases and 37 in controls did not have sexual dysfunction. $\mathrm{X}^{2}$ test was carried out and the difference was very highly statistically significant at 0.001 level. A very high frequency of sexual dysfunction in this group calls for added focus in to this aspect for a better quality of life for the patient. The relationship between locus of control, sexual dysfunction as assessed by FSFI and PANSS scores where higher scores denote a severe form of illness were subjected to co-relation analysis. Locus of control is identified to be external when the scores are higher than the cut-off point. CONCLUSION-Sexual dysfunction is one of the aspects which affect the overall quality of life and needs to be studied on a larger sample and the relationship between illness related, treatment related and environment related factors need to be studied. Individual disturbances in the various aspects of sexual dysfunction also need to be studied.
\end{abstract}

Keywords: Sexual Dysfunction, Females, Attribution, Schizophrenia, Prevalence

\section{Introduction}

Schizophrenia is an illness that may influence every aspect of the person's life [1]. Among the various domains affected by the illness, interpersonal relationships and sexual function need special attention. Sexual function is a complex human behavior that is affected by many physiological and psychological factors. Emotions for the respective partner and the relationship with partner have been identified as the strongest forecaster of sexual health. There are several factors, which play a pivotal role in females with sexual dysfunction in schizophrenia which includes stigma rejection, social isolation and inability to establish relationships. The reminiscence of the past sexual relationships like abusive hostile relationships and false expectations adversely affect sexual functioning. Rates of female sexual dysfunction in the general population was reported between 40 and 50\% [2], while in schizophrenia the rates reported have been between 30 and $80 \%$ [3]. A recent study by Singh et al 2009 reported female sexual dysfunction in $73.2 \%$ of females. Frequency of sexual dysfunction in 
women with schizophrenia in Asian countries has varied from 49.1\% in Japan (Fujii et al.2010) [4] to 52.6\% in Turkey [5]. There is a subtle conspiracy of silence and taboos that clouds the mentality of women where women hesitate to discuss sexuality openly [6]. Avasthi et al. in 2008 [7] conducted a study, where 100 consecutive women attending the Department of Pediatrics for the care of noncritical children in a tertiary care teaching hospital in Chandigarh. Sexual difficulties which were attributed to other factors were not considered significant enough to demand a thorough clinical assessment. A recent study from India witnessed sexual dysfunction among patients taking psychotropic using the UKU side effect rating scale ${ }^{1}$. Women (70.8\%) outmatched men (29\%) in frequency of sexual dysfunction. Amenorrhea, galactorrhea and decreased Sexual desire were the commonest sexual side effects [8]. However majority of the studies from India throws light only on male patients [9, $10,11]$. Though the treatment and the illness may influence the sexual functioning of women, the equation with the partner also plays an important role in sexual satisfaction. The quality of life between the patient and their partner is often disrupted due to mental illnesses [12] So this study throws a light to know about female sexual dysfunction and attribution in women with schizophrenia.

\subsection{Aim}

Prevalence of sexual dysfunction in females with and without schizophrenia.

\subsection{Objectives}

To assess the sexual functioning among females with schizophrenia and compare them with normal controls.

To identify the attributing factors of sexual dysfunction among females with schizophrenia.

\section{Method}

\subsection{Selection Criteria}

This is a cross sectional, hospital-based study, approved by the Institutional Ethics Committee (IEC) of SRM Medical college and health sciences. Women between the ages of 21 to 45 years, diagnosed with schizophrenia by ICD 10 criteria (World Health Organization, 1992) attending the outpatient services, who were under remission, and were in a marital relationship for at least six months were approached for the study. Remission was defined as a "state in which patients have experienced an improvement in core signs and symptoms to the extent that any remaining symptoms are of such low intensity that they no longer interfere significantly with behavior and are below the threshold typically utilized in justifying an initial diagnosis of schizophrenia"[12]. According to this criteria, the patient should score $<3$ in the following 3 items of positive symptoms of PANSS to fulfill remissions: delusions, hallucinatory behavior \& conceptual disorganization. Women with hypertension, Diabetes, Cardiac problems, severe dermatological problems, terminal illnesses, epilepsy, hysterectomy, menopausal women and hormonal therapy, substance abuse/Alcohol, Head injury were excluded from the study. Written informed consent was obtained from all the participants.

\subsection{Tools}

A semi-structured questionnaire was used to collect demographic details. GHQ12 known for good specificity and sensitivity was used to screen the patients for recognition and measurement of mental health.

Female sexual dysfunction was assessed using Female Sexual Function Index and Sexual attributes was calculated by Rotters Locus of control. Both these scales were translated in regional language (Tamil) by experts.

The Female Sexual Functioning Index (Rosen et al., 2000) is a brief (19 item) self-report measure used widely, that assesses sexual functioning among women in six separate dimensions (desire, arousal, lubrication, orgasm, satisfaction, pain). A score of 26.55 is taken as the cut off score for female sexual dysfunction. The Rotters locus of control is a 13 item questionnaire developed by Rotter (1966), that measures generalized expectancies for internal versus external control of reinforcement. A high score suggests External Locus of Control and a low score suggests Internal Locus of Control.

\section{Results}

A cohort of 50 women who had been on treatment for schizophrenia and in remission were compared with normal controls for sexual dysfunction and locus of control.

The mean age of the whole group was 33.19 with sd of + 5.93. age ranged from a minimum of 22 to a maximum of 44 . There was no statistically significant difference in age between cases and controls. Of the whole group 52 were housewives, 4 were working as cooks, 4 were shopkeepers, 2 were sweepers, 11 were students, 7 were teachers, 6 were bankers, 4 were accountants 4 were doctors and 6 were unemployed. Among the cases 35 were housewives, 5 teachers 4 bankers, 1 accountant 4 unemployed and one was a shopkeeper. Among controls 17 housewives 11 students 4 cooks 2 sweepers 3 accountants 2 teachers 3 shop keepers 2 bankers 4 were doctors and 2 were unemployed.

78 were from middle class background and 22 were from upper class. 13 had studied upto primary school, 60 up to secondary school while 13 had higher secondary level education, 7 were undergraduates and one person had post graduate education.

The groups were compared for sexual dysfunction. 45 women belonging to cases had sexual dysfunction as assessed by FSFI whilst 13 had had scored for sexual dysfunction is the control group. 5 in the cases and 37 in controls did not have sexual dysfunction. $\mathrm{X}^{2}$ test was carried out and the difference was very highly statistically significant at 0.001 level.

A very high frequency of sexual dysfunction in this group calls for added focus in to this aspect for a better quality of life for the patient. The relationship between locus of control, 
sexual dysfunction as assessed by FSFI and PANSS scores where higher scores denote a severe form of illness were subjected to co-relation analysis. Locus of control is identified to be external when the scores are higher than the cut-off point. Sexual dysfunction is considered to be present when the scores of FSFI is lower than the cut off point. When subjected to a co-relation analysis assuming that the scores in all these were continuous variables, there was a negative corelationship between locus of control and sexual dysfunction.
The relationship was statistically very significant at 0.0001 level negatively. This shows that those belonging to the cases (i-e) those who have illness exhibit external locus of control and they have significant sexual dysfunction. When sexual dysfunction was assessed for a co-relation against PANSS scores which measure psychopathology and sexual dysfunction there was a negative co-relationship showing that increased psychopathology does have an association with sexual dysfunction

Table 1. Descriptive analysis for age in study population $(N=100)$.

\begin{tabular}{llllllll}
\hline \multirow{2}{*}{ Parameter } & \multirow{2}{*}{ Mean \pm SD } & \multirow{2}{*}{ Median } & \multirow{2}{*}{ Min } & \multirow{2}{*}{ Max } & \multicolumn{2}{l}{$\mathbf{9 5 \%}$ C. I. for EXP (B) } \\
\cline { 5 - 8 } & & & & Lower & Upper & 34.37 \\
\hline Age & $33.19 \pm 5.93$ & 33.00 & 22.00 & 44.00 & 32.01 & 3 \\
\hline
\end{tabular}

The mean age was $33.19 \pm 5.93$ in the study population. Ranged between 22 years to 44 years (95\% CI 32.01 to 34.37 ) (Table 1)

Table 2. Descriptive analysis of study group in study population $(N=100)$.

\begin{tabular}{lll}
\hline Study group & Frequency & Percentage \\
\hline Schizophrenia & 50 & $50.00 \%$ \\
Normal control & 50 & $50.00 \%$ \\
\hline
\end{tabular}

Among the study population, $50(50 \%)$ were schizophrenia group and remaining $50(50 \%)$ were normal control group. (Table 2)

Table 3. Descriptive analysis of occupation in study population $(N=100)$.

\begin{tabular}{lll}
\hline Occupation & Frequency & Percent \\
\hline House wife & 52 & $52.00 \%$ \\
Cook & 4 & $4.00 \%$ \\
Shopkeeper & 4 & $4.00 \%$ \\
Sweeper & 2 & $2.00 \%$ \\
Student & 11 & $11.00 \%$ \\
Teacher & 7 & $7.00 \%$ \\
Banker & 6 & $6.00 \%$ \\
Accountant & 4 & $4.00 \%$ \\
Doctor & 4 & $4.00 \%$ \\
Unemployed & 6 & $6.00 \%$ \\
\hline
\end{tabular}

Table 4. Descriptive analysis of socio economic status in study population.

\begin{tabular}{lll}
\hline Socio Economic Status & Frequency & Percentage \\
\hline Middle Class & 78 & $78.00 \%$ \\
Upper Class & 22 & $22.00 \%$ \\
\hline
\end{tabular}

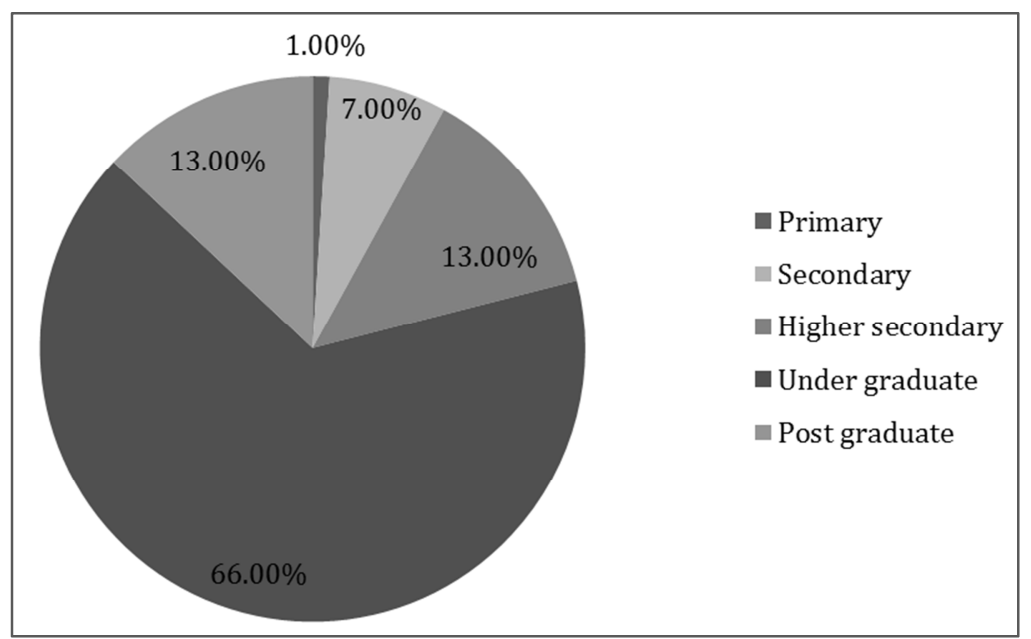

Figure 1. Pie chart of education in study population. 
Table 5. Comparison of study group with female sexual function index category of study population.

\begin{tabular}{|c|c|c|c|c|}
\hline \multirow{2}{*}{ Female Sexual Function Index category } & \multicolumn{2}{|l|}{ Group } & \multirow{2}{*}{ Chi square } & \multirow{2}{*}{ P-value } \\
\hline & Schizophrenia & Normal control & & \\
\hline $\begin{array}{l}\text { Sexual dysfunction } \\
\text { No sexual dysfunction }\end{array}$ & $\begin{array}{l}45(90 \%) \\
5(10 \%)\end{array}$ & $\begin{array}{l}13(26 \%) \\
37(74 \%)\end{array}$ & 42.03 & $<0.001$ \\
\hline
\end{tabular}

Table 6. Descriptive statistics among case and control groups.

\begin{tabular}{llll}
\hline & Mean & Standard Deviation & Normal \\
\hline Locus of control total score & 10.35 & 6.916 & 100 \\
Fsfi total score & 21.97 & 9.553 & 100 \\
\hline
\end{tabular}

Table 7. Correlation among case and control groups.

\begin{tabular}{llll}
\hline & & Locus of Control Total Score & Fsfi Total Score \\
\hline & Pearson correlation & 1 & $-.918^{* *}$ \\
Locus of control total score & SIG.(2-tailed) & & 0.000 \\
& N & 100 & 100 \\
Fsfi total score & Pearson corelation & $-.918^{* *}$ & 1 \\
& Sig $(2-$ tailed $)$ & 0.000 & 100 \\
\hline
\end{tabular}

Table 8. Descriptive Statistics of Case Group.

\begin{tabular}{llll}
\hline & Mean & Std. Deviation & N \\
\hline Locus of control total score & 14.76 & 5.847 & 50 \\
Fsfi total score & 15.70 & 7.916 & 50 \\
Panss total score & 40.54 & 4.287 & 50 \\
\hline
\end{tabular}

Table 9. Correlation of Variables of case Group.

\begin{tabular}{|c|c|c|c|c|}
\hline & & Locus of control total score & Fsfi total score & Panss total score \\
\hline \multirow{3}{*}{ Locus of control total score } & Pearson correlation & 1 & $-.885^{* *}$ & $.324 *$ \\
\hline & $\operatorname{Sig}(2-$ tailed $)$ & & 000 & 022 \\
\hline & $\mathrm{N}$ & 50 & 50 & 50 \\
\hline \multirow{3}{*}{ Fsfi total score } & Pearson correlation & $-.885^{* *}$ & 1 & $-.370 * *$ \\
\hline & Sig (2-tailed) & .000 & & .008 \\
\hline & $\mathrm{N}$ & 50 & 50 & 50 \\
\hline \multirow{3}{*}{ Panss total score } & Pearson correlation & $324 *$ & $-.370 * *$ & 1 \\
\hline & Sig (2 tailed) & .022 & .008 & \\
\hline & & 50 & 50 & 50 \\
\hline
\end{tabular}

**Correlation is significant at the 0.01 level (2 tailed).

*correlation is significant at the 0.05 level ( 2 tailed).

\section{Discussion}

Though causes of sexual dysfunction are multifactorial, illness related variables do play an important part. A very high frequency of sexual dysfunction in this group calls for added focus in to this aspect for a better quality of life for the patient. Sexual dysfunction impacts the marital relationship and sometimes strained marital relationship can also be a factor in causing or being associated with sexual dysfunction. Regardless of it being a cause or effect, addressing this issue is possible and is within the professional responsibility of the clinician. Use of a standardized tool is an important factor in this study which objectively measures the dysfunction. Simiyon et al in a similar study have identified sexual dysfunction in $44 \%$ of the cases where as in this study it appears to be in $90 \%$ of the cases. The wide difference cannot be explained by difference in location as both the studies are carried out in a similar cultural and geographical background. Further assessments and studies are needed to explain the huge difference. Ucok's study among schizophrenic women on medications, who were assessed for psychopathology and side effects co-related with sexual dysfunction, reports that $52.6 \%$ had sexual dysfunction. A similar study by Shetageri et al showed an incidence of $68.32 \%$ had sexual dysfunction. As reported by Baggaley, Systematic reviews have shown that sexual dysfunction is seen in $30-80 \%$ of the women with psychotic illness with or without treatment.

The relationship between locus of control, sexual dysfunction as assessed by FSFI and PANSS scores; where higher scores denote a severe form of illness were subjected to Correlation analysis. Locus of control is identified to be external when the scores are higher than the cut-off point. Sexual dysfunction is considered to be present when the scores of FSFI is lower than the cut off point. When subjected to a co-relation analysis assuming that the scores in all these were continuous variables, there was a negative corelationship between locus of control and sexual dysfunction. The relationship was statistically very significant at 0.0001 
level negatively. This shows that those belonging to the cases (i-e) those who have illness exhibit external locus of control and have significant sexual dysfunction.

When sexual dysfunction was assessed for a correlation against PANSS scores which measure psychopathology and sexual dysfunction there was a negative correlation showing that increased psychopathology does have an association with sexual dysfunction. Simiyon et al have studied the relationship between psychopathology and sexual dysfunction and have come to a similar conclusion.

Sexual dysfunction in this study appears to be correlated with severity of psychopathology as assessed by PANSS scale and the locus of control as assessed by Rotter's locus of control scale. Sexual dysfunction impacts on the quality of life along with interpersonal relationship with the partner. Women have a comparatively less say in matters affecting them and presence of a chronic illness coupled with medication side effects can affect the quality of life by themselves apart from impacting on sexual function also. It might improve the quality of life in a woman if her sexual function improves.

\section{Conclusion}

Sexual dysfunction is one of the aspects which affect the overall quality of life and needs to be studied on a larger sample and the relationship between illness related, treatment related and environment related factors need to be studied. Separate analysis in all the domains of female sexual functioning index to be done to know which domain is commonly affected. Future studies can focus on comparing prevalence of sexual dysfunction between schizophrenia and other mental illnesses to get a clear picture of the severity in each mental illness.

\section{References}

[1] M. Simiyon et al, Sexual dysfunction among women with Schizophrenia-A cross sectional study from India, Asian Journal of Psychiatry AJP 24 (2016) 93-98

[2] McCabe, M. P., Sharlip, I. D., Lewis, R., Atalla, E., Balon, R., Fisher, A. D., Laumann, E., Lee, S. W., Segraves, R. T., 2016b. Incidence and Prevalence of Sexual Dysfunction in Women and Men: A Consensus Statement from the Fourth International Consultation on Sexual Medicine 2015Incidence and prevalence of sexual dysfunction in women and men: a consensus statement from the fourth international consultation on sexual medicine 2015. J. Sex. Med. 13, 144-152. doi:http://dx.doi.org/10.1016/j.jsxm.2015.12.034
[3] Baggaley, M., 2008. Sexual dysfunction in schizophrenia: focus on recent evidence. Hum. Psychopharmacol. 23, 201209. doi:http://dx.doi.org/10.1002/hup.924

[4] Fujii, A., Yasui-Furukori, N., Sugawara, N., Sato, Y., Nakagami, T., Saito, M., Kaneko, S., 2010. Sexual dysfunction in Japanese patients with schizophrenia treated with antipsychotics. Prog. Neuropsychopharmacol. Biol. Psychiatry 34, 288-293. doi: http://dx.doi.org/10.1016/j.pnpbp.2009.11.022

[5] Üçok, A., _Incesu, C., Aker, T., Erkoç, Ş., 2007. Sexual dysfunction in patients with schizophrenia on antipsychotic medication. Eur. Psychiatry 22, 328-333. doi: http://dx.doi.org/10.1016/j.eurpsy.2007.01.001

[6] Varghese, K. M., Bansal, R., Kekre, A. N., Jacob, K. S., 2012. Sexual dysfunction among young married women in southern India. Int. Urogynecol. J. 23, 1771-1774. doi: http://dx.doi.org/10.1007/s00192-012-1782-3

[7] Ajit avasthi et al., Sexual Behavior of Married Young Women: A Preliminary Study from North India. Indian J Community Med. 2008 Jul; 33(3): 163-167.

[8] Lucca, J., Ramesh, M., Ram, D., Kurian, J., Mathew, N., 2016. Psychotropic medication-induced sexual dysfunction and its interference with patient0s daily performance: a crosssectional study. Egypt. J. Psychiatry 37, 36. doi:http://dx. doi.org/10.4103/1110-1105.180268

[9] Nagaraj, A. K. M., Pai, N. B., Rao, S., 2009. A comparative study of sexual dysfunction involving risperidone, quetiapine, and olanzapine. Indian J. Psychiatry 51, 265-271. doi:http://dx.doi.org/10.4103/0019-5545.58291

[10] Nebhinani, N., Grover, S., Avasthi, A., 2012. Sexual dysfunction in male subjects receiving trifluoperazine, risperidone, or olanzapine: rates vary with assessment questionnaire. Sexual dysfunction in male subjects receiving trifluoperazine, risperidone, or olanzapine: rates vary with assessment questionnaire. Prim. Care Companion CNS Disord. 14 doi:http://dx.doi.org/ 10.4088/pcc.11m01199

[11] Thangadurai, P., Gopalakrishnan, R., Kuruvilla, A., Jacob, K. S., Abraham, V. J., Prasad, J., 2014. Sexual dysfunction among men in secondary care in southern India: Nature, prevalence, clinical features and explanatory modelsSexual dysfunction among men in secondary care in southern India: nature, prevalence, clinical features and explanatory models. Natl. Med. J. (India) 27, 198-201.

[12] Andreasen, N. C., Carpenter, W. T., Kane, J. M., Lasser, R. A., Marder, S. R., Weinberger, D. R., 2005. Remission in schizophrenia: proposed criteria and rationale for consensus. Am. J. Psychiatry 162, 441-449. doi:http://dx.doi.org/10.1176/appi. ajp.162.3.441 